Article

\title{
Multiple Solutions for Partial Discrete Dirichlet Problems Involving the $p$-Laplacian
}

\author{
Sijia Du ${ }^{1,2}$ and Zhan Zhou ${ }^{1,2, *}$ \\ 1 School of Mathematics and Information Science, Guangzhou University, Guangzhou 510006, China; \\ qingzhoujiangxue@126.com \\ 2 Center for Applied Mathematics, Guangzhou University, Guangzhou 510006, China \\ * Correspondence: zzhou@gzhu.edu.cn
}

Received: 18 October 2020; Accepted: 12 November 2020; Published: 14 November 2020

\begin{abstract}
Due to the applications in many fields, there is great interest in studying partial difference equations involving functions with two or more discrete variables. In this paper, we deal with the existence of infinitely many solutions for a partial discrete Dirichlet boundary value problem with the $p$-Laplacian by using critical point theory. Moreover, under appropriate assumptions on the nonlinear term, we determine open intervals of the parameter such that at least two positive solutions and an unbounded sequence of positive solutions are obtained by using the maximum principle. We also show two examples to illustrate our results.
\end{abstract}

Keywords: partial difference equations; boundary value problem; $p$-laplacian; multiple solutions; critical point theory

\section{Introduction}

Let $\mathbb{Z}, \mathbb{R}, \mathbb{N}$ denote all integers, real numbers and positive integers, respectively. Define $\mathbb{Z}(a, b)=$ $\{a, a+1, \cdots, b\}$ for any $a, b \in \mathbb{Z}$ with $a \leq b$.

In this paper, we consider the following problem, namely $\left(S_{\lambda}^{f}\right)$

$$
\Delta_{1}\left(\phi_{p}\left(\Delta_{1} x(i-1, j)\right)\right)+\Delta_{2}\left(\phi_{p}\left(\Delta_{2} x(i, j-1)\right)\right)+\lambda f((i, j), x(i, j))=0,(i, j) \in \mathbb{Z}(1, m) \times \mathbb{Z}(1, n),
$$

with boundary conditions

$$
\begin{aligned}
& x(i, 0)=x(i, n+1)=0, \quad i \in \mathbb{Z}(0, m+1), \\
& x(0, j)=x(m+1, j)=0, \quad j \in \mathbb{Z}(0, n+1),
\end{aligned}
$$

where $m$ and $n$ are given positive integers, $\lambda$ is a positive real parameter, $\Delta_{1}$ and $\Delta_{2}$ are forward difference operators, respectively defined by $\Delta_{1} x(i, j)=x(i+1, j)-x(i, j)$ and $\Delta_{2} x(i, j)=x(i, j+1)-x(i, j), \Delta_{1}^{2} x(i, j)=\Delta_{1}\left(\Delta_{1} x(i, j)\right)$ and $\Delta_{2}^{2} x(i, j)=\Delta_{2}\left(\Delta_{2} x(i, j)\right), \phi_{p}$ is the $p$-Laplacian operator given by $\phi_{p}(s)=|s|^{p-2} s, 1<p<+\infty$ and $f((i, j), \cdot) \in C(\mathbb{R}, \mathbb{R})$ for all $(i, j) \in \mathbb{Z}(1, m) \times \mathbb{Z}(1, n)$.

The study of difference equations has captured special attention, which is due to the fact that difference equations are widely used as mathematical models in discrete optimization, physics, population genetics, etc. [1-4]. Many researchers have done in-depth study on the difference equation and use critical point theory to acquire some wonderful conclusions. For example, some results on homoclinic solutions [5-13], periodic solutions [14-16], ground state solutions [17,18] and solutions for boundary value problems [19-29] have been achieved. Especially, in recent years, owing to more and more applications of partial difference equation mathematical models in many fields, such as economy, 
computer science and control systems, there has aroused a great deal of interest in studying partial difference equations involving functions with two or more discrete variables. With the increase of research, many results have been obtained [30-32].

In [30], Shapour Heidarkhani and Maurizio Imbesi considered the following partial discrete Dirichlet problem $\left(E_{\lambda}^{f}\right)$

$$
\Delta_{1}^{2} u(i-1, j)+\Delta_{2}^{2} u(i, j-1)+\lambda f((i, j), u(i, j))=0, \quad(i, j) \in \mathbb{Z}(1, m) \times \mathbb{Z}(1, n),
$$

with boundary conditions

$$
\begin{aligned}
& u(i, 0)=u(i, n+1)=0, \quad i \in \mathbb{Z}(1, m), \\
& u(0, j)=u(m+1, j)=0, \quad j \in \mathbb{Z}(1, n) .
\end{aligned}
$$

The authors transformed the matrix form into the one-dimensional vector form, and obtained the existence of at least three solutions for problem $\left(E_{\lambda}^{f}\right)$ by utilizing two critical point theorems.

In [31], Marek Galewski and Aleksandra Orpel obtained some existence results of $\left(E_{\lambda}^{f}\right)$ in light of variational methods and some monotonicity results.

Maurizio Imbesi and Giovanni Molica Bisci [32] determined unbounded intervals of parameters such that $\left(E_{\lambda}^{f}\right)$ admitted either an unbounded sequence of solutions or a pairwise distinct sequence of solutions by the critical point theory.

However, until now, there is very little research on the partial difference equations with the $p$-Laplacian. For this reason, this paper is to study the existence of multiple solutions for partial discrete Dirichlet problems involving the $p$-Laplacian. In this paper, in the framework of variational methods, we consider the two-dimensional discrete problem $\left(S_{\lambda}^{f}\right)$ by using critical point theory and we come up with more specific sets of parameters such that the existence of infinitely many solutions for problem $\left(S_{\lambda}^{f}\right)$ can be obtained. Under some proper assumptions, we deal with the existence of multiple solutions of problem $\left(S_{\lambda}^{f}\right)$ by applying Theorem 3.3 of [33] in Theorem 2. Furthermore, we show that problem $\left(S_{\lambda}^{f}\right)$ admits at least two positive solutions in Theorem 3. In addition, we obtain that problem $\left(S_{\lambda}^{f}\right)$ admits an unbounded sequence of solutions by utilizing Theorem 2.1 of [34] in Theorem 4.

The structure of the rest of this paper is as follows. In Section 2, some basic lemmas and propositions are showed. In Section 3, we give our main results. In Section 4, two examples are presented to explicate our results. We conclude our results in the last section.

\section{Preliminaries}

Let $E$ denote a finite dimensional real Banach space and let $I_{\lambda}: E \rightarrow \mathbb{R}$ be a function satisfying the following structure hypothesis:

(A) $I_{\lambda}(x):=\Phi(x)-\lambda \Psi(x)$ for all $x \in E$, where $\Phi, \Psi: E \rightarrow \mathbb{R}$ are two functions of class $C^{1}$ on $E$ with $\Phi$ coercive, i.e., $\lim _{\|x\| \rightarrow \infty} \Phi(x)=+\infty$, and $\lambda$ is a real positive parameter.

The following lemma comes from Theorem 2.2 of [30].

Lemma 1. Assume that the condition $(A)$ holds. We have

(B) $\Phi$ is convex and $\inf _{E} \Phi=\Phi(0)=\Psi(0)=0$;

(C) for each $\lambda>0$ and for every $x_{1}, x_{2} \in E$ which are local minima for the functional $I_{\lambda}:=\Phi-\lambda \Psi$ and such that $\Psi\left(x_{1}\right) \geq 0$ and $\Psi\left(x_{2}\right) \geq 0$, one has $\inf _{0 \leq t \leq 1} \Psi\left(t x_{1}+(1-t) x_{2}\right) \geq 0$.

Further, assume that there are two positive constants $\rho_{1}, \rho_{2}$ and $u \in E$, with $2 \rho_{1}<\Phi(u)<\frac{\rho_{2}}{2}$, such that $\left(a_{1}\right)$

$$
\frac{\sup _{x \in \Phi^{-1}\left(-\infty, \rho_{1}\right)} \Psi(x)}{\rho_{1}}<\frac{2}{3} \cdot \frac{\Psi(u)}{\Phi(u)}
$$


$\left(a_{2}\right)$

$$
\frac{\sup _{x \in \Phi^{-1}\left(-\infty, \rho_{2}\right)} \Psi(x)}{\rho_{2}}<\frac{1}{3} \cdot \frac{\Psi(u)}{\Phi(u)} .
$$

Then, for each $\lambda \in\left(\frac{3}{2} \cdot \frac{\Phi(u)}{\Psi(u)}, \min \left\{\frac{\rho_{1}}{\sup _{x \in \Phi^{-1}\left(-\infty, \rho_{1}\right)} \Psi(x)}, \frac{\rho_{2} / 2}{\sup _{x \in \Phi^{-1}\left(-\infty, \rho_{2}\right)} \Psi(x)}\right\}\right)$, the functional $I_{\lambda}$ has at least three distinct critical points which lie in $\Phi^{-1}\left(-\infty, \rho_{2}\right)$.

The following lemma comes from Corollary 3.1 of [33].

Lemma 2. Assume that the condition $(A)$ holds. We have

(D) $\Phi$ is convex and $\inf _{E} \Phi=\Phi(0)=\Psi(0)=0$;

(E) for each $\lambda>0$ and for every $x_{1}, x_{2} \in E$ which are local minima for the functional $I_{\lambda}:=\Phi-\lambda \Psi$ and such that $\Psi\left(x_{1}\right) \geq 0$ and $\Psi\left(x_{2}\right) \geq 0$, one has $\inf _{0 \leq t \leq 1} \Psi\left(t x_{1}+(1-t) x_{2}\right) \geq 0$.

Further, assume that there are two positive constants $\rho_{1}, \rho_{2}$ and $u \in E$, with $\rho_{1}<\Phi(u)<\frac{\rho_{2}}{2}$, such that $\left(a_{3}\right)$

$$
\frac{\sup _{x \in \Phi^{-1}\left(-\infty, \rho_{1}\right)} \Psi(x)}{\rho_{1}}<\frac{1}{2} \cdot \frac{\Psi(u)}{\Phi(u)}
$$

$\left(a_{4}\right)$

$$
\sup _{\frac{x \in \Phi^{-1}\left(-\infty, \rho_{2}\right)}{\rho_{2}} \Psi(x)}<\frac{1}{4} \cdot \frac{\Psi(u)}{\Phi(u)} .
$$

Then, for each $\lambda \in\left(\frac{2 \Phi(u)}{\Psi(u)}, \min \left\{\frac{\rho_{1}}{\sup _{x \in \Phi^{-1}\left(-\infty, \rho_{1}\right)} \Psi(x)}, \frac{\rho_{2} / 2}{\sup _{x \in \Phi^{-1}\left(-\infty, \rho_{2}\right)} \Psi(x)}\right\}\right)$, the functional $I_{\lambda}$ has at least three distinct critical points which lie in $\Phi^{-1}\left(-\infty, \rho_{2}\right)$.

Let $t, t_{1}, t_{2}>\inf _{E} \Phi$ with $t_{2}>t_{1}$ and $t_{3}>0$ such that

$$
\begin{aligned}
& \varphi(t)=\inf _{x \in \Phi^{-1}(-\infty, t)} \frac{\left(\sup _{x \in \Phi^{-1}(-\infty, t)} \Psi(x)\right)-\Psi(x)}{t-\Phi(x)}, \\
& \beta\left(t_{1}, t_{2}\right)=\inf _{x \in \Phi^{-1}\left(-\infty, t_{1}\right)} \sup _{y \in \Phi^{-1}\left[t_{1}, t_{2}\right)} \frac{\Psi(y)-\Psi(x)}{\Phi(y)-\Phi(x)}, \\
& \gamma\left(t_{2}, t_{3}\right)=\frac{\sup _{x \in \Phi^{-1}\left(-\infty, t_{2}+t_{3}\right)} \Psi(x)}{t_{3}}, \\
& \alpha\left(t_{1}, t_{2}, t_{3}\right)=\max \left\{\varphi\left(t_{1}\right), \varphi\left(t_{2}\right), \gamma\left(t_{2}, t_{3}\right)\right\} .
\end{aligned}
$$

The following lemma comes from Theorem 3.3 of [33].

Lemma 3. Assume that the condition $(A)$ holds. We have

$(F) \Phi$ is convex and $\inf _{E} \Phi=\Phi(0)=\Psi(0)=0$;

(G) for each $\lambda>0$ and for every $x_{1}, x_{2} \in E$ which are local minima for the functional $I_{\lambda}:=\Phi-\lambda \Psi$ and such that $\Psi\left(x_{1}\right) \geq 0$ and $\Psi\left(x_{2}\right) \geq 0$, one has $\inf _{0 \leq s \leq 1} \Psi\left(s x_{1}+(1-s) x_{2}\right) \geq 0$. 
Further, assume that there are three positive constants $\rho_{1}, \rho_{2}, \rho_{3}$ with $\rho_{1}<\rho_{2}$, such that

$\left(a_{5}\right) \varphi\left(\rho_{1}\right)<\beta\left(\rho_{1}, \rho_{2}\right)$;

$\left(a_{6}\right) \varphi\left(\rho_{2}\right)<\beta\left(\rho_{1}, \rho_{2}\right)$;

$\left(a_{7}\right) \gamma\left(\rho_{2}, \rho_{3}\right)<\beta\left(\rho_{1}, \rho_{2}\right)$.

Then, for each $\lambda \in\left(\frac{1}{\beta\left(\rho_{1}, \rho_{2}\right)}, \frac{1}{\alpha\left(\rho_{1}, \rho_{2}, \rho_{3}\right)}\right)$, the functional $I_{\lambda}$ has three distinct critical points.

Let

$$
\gamma=\liminf _{t \rightarrow+\infty} \varphi(t), \quad \delta=\liminf _{t \rightarrow\left(\inf _{E} \Phi\right)^{+}} \varphi(t) .
$$

Clearly, $\gamma \geq 0$ and $\delta \geq 0$. When $\gamma=0$ (or $\delta=0$ ), in the sequel, we agree to read $\frac{1}{\gamma}$ (or $\frac{1}{\delta}$ ) as $+\infty$. The following lemma comes from Theorem 2.1 of [34].

Lemma 4. Assume that the condition (A) holds, one has

(i) If $\gamma<+\infty$ then, for each $\lambda \in\left(0, \frac{1}{\gamma}\right)$, the following alternative holds: either

$\left(i_{1}\right) I_{\lambda}$ possesses a global minimum, or

$\left(i_{2}\right)$ there is a sequence $\left\{x_{k}\right\}$ of critical points (local minima) of $I_{\lambda}$ such that $\lim _{k \rightarrow+\infty} \Phi\left(x_{k}\right)=+\infty$.

(j) If $\delta<+\infty$ then, for each $\lambda \in\left(0, \frac{1}{\delta}\right)$, the following alternative holds: either

$\left(j_{1}\right)$ there is a global minimum of $\Phi$ which is a local minimum of $I_{\lambda}$, or

$\left(j_{2}\right)$ there is a sequence $\left\{x_{k}\right\}$ of pairwise distinct critical points (local minima) of $I_{\lambda}$, with $\lim _{k \rightarrow+\infty} \Phi\left(x_{k}\right)=\inf _{E} \Phi$, which weakly converges to a global minimum of $\Phi$.

Now we consider the mn-dimensional Banach space

$$
\begin{gathered}
S=\{x: \quad \mathbb{Z}(0, m+1) \times \mathbb{Z}(0, n+1) \rightarrow \mathbb{R} \text { such that } x(i, 0)=x(i, n+1)=0, \\
i \in \mathbb{Z}(0, m+1) \text { and } x(0, j)=x(m+1, j)=0, j \in \mathbb{Z}(0, n+1)\},
\end{gathered}
$$

endowed with the norm

$$
\|x\|=\left(\sum_{j=1}^{n} \sum_{i=1}^{m+1}\left|\Delta_{1} x(i-1, j)\right|^{p}+\sum_{i=1}^{m} \sum_{j=1}^{n+1}\left|\Delta_{2} x(i, j-1)\right|^{p}\right)^{\frac{1}{p}}, x \in S .
$$

For each $x \in S$, let

$$
\begin{gathered}
\Phi(x)=\sum_{j=1}^{n} \sum_{i=1}^{m+1} \frac{1}{p}\left|\Delta_{1} x(i-1, j)\right|^{p}+\sum_{i=1}^{m} \sum_{j=1}^{n+1} \frac{1}{p}\left|\Delta_{2} x(i, j-1)\right|^{p}, \\
\Psi(x)=\sum_{j=1}^{n} \sum_{i=1}^{m} F((i, j), x(i, j)),
\end{gathered}
$$

where $F((i, j), x)=\int_{0}^{x} f((i, j), \xi) d \xi$ for every $((i, j), x) \in \mathbb{Z}(1, m) \times \mathbb{Z}(1, n) \times \mathbb{R}$.

Define

$$
I_{\lambda}(x)=\Phi(x)-\lambda \Psi(x),
$$

for any $x \in S$. It is clear that $I_{\lambda} \in C^{1}(S, \mathbb{R})$ with 


$$
\begin{aligned}
\Phi^{\prime}(x)(z)= & \lim _{t \rightarrow 0} \frac{\Phi(x+t z)-\Phi(x)}{t} \\
= & \sum_{j=1}^{n} \sum_{i=1}^{m+1} \phi_{p}\left(\Delta_{1} x(i-1, j)\right) \Delta_{1} z(i-1, j)+\sum_{i=1}^{m} \sum_{j=1}^{n+1} \phi_{p}\left(\Delta_{2} x(i, j-1)\right) \Delta_{2} z(i, j-1) \\
= & \sum_{j=1}^{n} \sum_{i=1}^{m} \phi_{p}\left(\Delta_{1} x(i-1, j)\right) \Delta_{1} z(i-1, j)-\sum_{j=1}^{n} \phi_{p}\left(\Delta_{1} x(m, j)\right) z(m, j) \\
& +\sum_{i=1}^{m} \sum_{j=1}^{n} \phi_{p}\left(\Delta_{2} x(i, j-1)\right) \Delta_{2} z(i, j-1)-\sum_{i=1}^{m} \phi_{p}\left(\Delta_{2} x(i, n)\right) z(i, n) \\
= & -\sum_{j=1}^{n} \sum_{i=1}^{m} \Delta_{1} \phi_{p}\left(\Delta_{1} x(i-1, j)\right) z(i, j)-\sum_{i=1}^{m} \sum_{j=1}^{n} \Delta_{2} \phi_{p}\left(\Delta_{2} x(i, j-1)\right) z(i, j)
\end{aligned}
$$

and

$$
\Psi^{\prime}(x)(z)=\lim _{t \rightarrow 0} \frac{\Psi(x+t z)-\Psi(x)}{t}=\sum_{j=1}^{n} \sum_{i=1}^{m} f((i, j), x(i, j)) z(i, j)
$$

for all $x, z \in S$.

Now

$$
\begin{aligned}
{\left[\Phi^{\prime}(x)-\lambda \Psi^{\prime}(x)\right](z)=} & -\sum_{j=1}^{n} \sum_{i=1}^{m}\left[\Delta_{1} \phi_{p}\left(\Delta_{1} x(i-1, j)\right)+\Delta_{2} \phi_{p}\left(\Delta_{2} x(i, j-1)\right)\right. \\
& +\lambda f((i, j), x(i, j))] z(i, j) .
\end{aligned}
$$

Consequently, the critical points of $I_{\lambda}$ in $S$ are exactly the solutions of problem $\left(S_{\lambda}^{f}\right)$.

Proposition 1. For every $x \in S$, the relation

$$
\max _{\substack{i \in \mathbb{Z}(1, m) \\ j \in \mathbb{Z}(1, n)}}\{|x(i, j)|\} \leq \frac{(m+n+2)^{\frac{p-1}{p}}}{4}\|x\|
$$

holds.

Proof. For any given $x \in S$, there exist $s \in \mathbb{Z}(1, m)$ and $\tau \in \mathbb{Z}(1, n)$ such that

$$
|x(s, \tau)|=\max _{\substack{i \in \mathbb{Z}(1, m) \\ j \in \mathbb{Z}(1, n)}}\{|x(i, j)|\}
$$

Since $x(i, 0)=x(i, n+1)=0, i \in \mathbb{Z}(0, m+1)$ and $x(0, j)=x(m+1, j)=0, j \in \mathbb{Z}(0, n+1)$, we can obtain

$$
\begin{aligned}
|x(s, \tau)| & =\frac{1}{2}\left|\sum_{i=1}^{s} \Delta_{1} x(i-1, \tau)+\sum_{j=1}^{\tau} \Delta_{2} x(s, j-1)\right| \\
& \leq \frac{1}{2} \sum_{i=1}^{s}\left|\Delta_{1} x(i-1, \tau)\right|+\frac{1}{2} \sum_{j=1}^{\tau}\left|\Delta_{2} x(s, j-1)\right| \\
& \leq \frac{1}{2} \cdot(s+\tau)^{\frac{1}{q}}\left(\sum_{i=1}^{s}\left|\Delta_{1} x(i-1, \tau)\right|^{p}+\sum_{j=1}^{\tau}\left|\Delta_{2} x(s, j-1)\right|^{p}\right)^{\frac{1}{p}}
\end{aligned}
$$


and

$$
\begin{aligned}
|x(s, \tau)| & =\frac{1}{2}\left|\sum_{i=s+1}^{m+1} \Delta_{1} x(i-1, \tau)+\sum_{j=\tau+1}^{n+1} \Delta_{2} x(s, j-1)\right| \\
& \leq \frac{1}{2} \sum_{i=s+1}^{m+1}\left|\Delta_{1} x(i-1, \tau)\right|+\frac{1}{2} \sum_{j=\tau+1}^{n+1}\left|\Delta_{2} x(s, j-1)\right| \\
& \leq \frac{1}{2} \cdot(m+n-s-\tau+2)^{\frac{1}{q}} \cdot\left(\sum_{i=s+1}^{m+1}\left|\Delta_{1} x(i-1, \tau)\right|^{p}+\sum_{j=\tau+1}^{n+1}\left|\Delta_{2} x(s, j-1)\right|^{p}\right)^{\frac{1}{p}},
\end{aligned}
$$

where $q$ is the conjugative number of $p$, that is, $\frac{1}{p}+\frac{1}{q}=1$. If

$$
\begin{aligned}
& \sum_{i=1}^{s}\left|\Delta_{1} x(i-1, \tau)\right|^{p}+\sum_{j=1}^{\tau}\left|\Delta_{2} x(s, j-1)\right|^{p} \\
\leq & \frac{(m+n+2)^{p-1}}{2^{p} \cdot(s+\tau)^{p-1}} \cdot\left(\sum_{i=1}^{m+1}\left|\Delta_{1} x(i-1, \tau)\right|^{p}\right) \\
& +\frac{(m+n+2)^{p-1}}{2^{p} \cdot(s+\tau)^{p-1}} \cdot\left(\sum_{j=1}^{n+1}\left|\Delta_{2} x(s, j-1)\right|^{p}\right)
\end{aligned}
$$

then we can get

$$
\max _{\substack{i \in \mathbb{Z}(1, m) \\ j \in \mathbb{Z}(1, n)}}\{|x(i, j)|\} \leq \frac{(m+n+2)^{\frac{p-1}{p}}}{4}\left(\sum_{i=1}^{m+1}\left|\Delta_{1} x(i-1, \tau)\right|^{p}+\sum_{j=1}^{n+1}\left|\Delta_{2} x(s, j-1)\right|^{p}\right)^{\frac{1}{p}} .
$$

So, we obtain the required relation (1). If, on the contrary,

$$
\begin{aligned}
\sum_{i=1}^{s}\left|\Delta_{1} x(i-1, \tau)\right|^{p}+\sum_{j=1}^{\tau}\left|\Delta_{2} x(s, j-1)\right|^{p}> & \frac{(m+n+2)^{p-1}}{2^{p} \cdot(s+\tau)^{p-1}} \cdot\left(\sum_{i=1}^{m+1}\left|\Delta_{1} x(i-1, \tau)\right|^{p}\right) \\
& +\frac{(m+n+2)^{p-1}}{2^{p} \cdot(s+\tau)^{p-1}} \cdot\left(\sum_{j=1}^{n+1}\left|\Delta_{2} x(s, j-1)\right|^{p}\right),
\end{aligned}
$$

then we have

$$
\begin{aligned}
& \sum_{i=s+1}^{m+1}\left|\Delta_{1} x(i-1, \tau)\right|^{p}+\sum_{j=\tau+1}^{n+1}\left|\Delta_{2} x(s, j-1)\right|^{p} \\
= & \sum_{i=1}^{m+1}\left|\Delta_{1} x(i-1, \tau)\right|^{p}+\sum_{j=1}^{n+1}\left|\Delta_{2} x(s, j-1)\right|^{p} \\
& -\left(\sum_{i=1}^{s}\left|\Delta_{1} x(i-1, \tau)\right|^{p}+\sum_{j=1}^{\tau}\left|\Delta_{2} x(s, j-1)\right|^{p}\right) \\
< & \left(1-\frac{(m+n+2)^{p-1}}{2^{p} \cdot(s+\tau)^{p-1}}\right) \cdot\left(\sum_{i=1}^{m+1}\left|\Delta_{1} x(i-1, \tau)\right|^{p}\right) \\
& +\left(1-\frac{(m+n+2)^{p-1}}{2^{p} \cdot(s+\tau)^{p-1}}\right) \cdot\left(\sum_{j=1}^{n+1}\left|\Delta_{2} x(s, j-1)\right|^{p}\right) .
\end{aligned}
$$

Moreover, we have

$$
\begin{aligned}
& |x(s, \tau)| \\
< & \frac{1}{2} \cdot(m+n-s-\tau+2)^{\frac{1}{q}} \cdot\left(1-\frac{(m+n+2)^{p-1}}{2^{p} \cdot(s+\tau)^{p-1}}\right)^{\frac{1}{p}} \cdot\left(\sum_{i=1}^{m+1}\left|\Delta_{1} x(i-1, \tau)\right|^{p}+\sum_{j=1}^{n+1}\left|\Delta_{2} x(s, j-1)\right|^{p}\right)^{\frac{1}{p}} .
\end{aligned}
$$

We claim that inequality

$$
\frac{1}{2} \cdot(m+n-s-\tau+2)^{\frac{1}{q}} \cdot\left(1-\frac{(m+n+2)^{p-1}}{2^{p} \cdot(s+\tau)^{p-1}}\right)^{\frac{1}{p}} \leq \frac{(m+n+2)^{\frac{1}{q}}}{4}
$$


holds. In fact, we define a function $g:(0, m+n+2) \rightarrow \mathbb{R}$ by

$$
g(t)=\frac{1}{(m+n-t+2)^{p-1}}+\frac{1}{t^{p-1}} .
$$

The function $g$ can attain its minimum $\frac{2^{p}}{(m+n+2)^{p-1}}$ at $t=\frac{m+n+2}{2}$. Since $s \in \mathbb{Z}(1, m), \tau \in \mathbb{Z}(1, n)$, we can get $g(s+\tau) \geq \frac{2^{p}}{(m+n+2)^{p-1}}$, that is,

$$
\frac{1}{(m+n-s-\tau+2)^{p-1}}+\frac{1}{(s+\tau)^{p-1}} \geq \frac{2^{p}}{(m+n+2)^{p-1}} .
$$

This implies assertion (2) and we can obtain the required inequality (1). The proof is complete.

Remark 1. Obviously, when $m=1$ and $n=1$, then $\|x\|=4^{\frac{1}{p}}|x(1,1)|$ and the inequality in (1) holds.

Now we establish the strong maximum principle for problem $\left(S_{\lambda}^{f}\right)$.

Proposition 2. Assume that there exists $\bar{x}: \mathbb{Z}(0, m+1) \times \mathbb{Z}(0, n+1) \rightarrow \mathbb{R}$ such that

$$
\Delta_{1}\left(\phi_{p}\left(\Delta_{1} \bar{x}(i-1, j)\right)\right)+\Delta_{2}\left(\phi_{p}\left(\Delta_{2} \bar{x}(i, j-1)\right)\right) \leq 0,
$$

for every $(i, j) \in \mathbb{Z}(1, m) \times \mathbb{Z}(1, n)$ and

$$
\bar{x}(i, 0)=\bar{x}(i, n+1)=0, \quad i \in \mathbb{Z}(0, m+1), \quad \bar{x}(0, j)=\bar{x}(m+1, j)=0, \quad j \in \mathbb{Z}(0, n+1),
$$

then, either $\bar{x}$ is the identically zero function or $\bar{x}(i, j)>0$ for every $(i, j) \in \mathbb{Z}(1, m) \times \mathbb{Z}(1, n)$.

Note that when $f: \mathbb{Z}(1, m) \times \mathbb{Z}(1, n) \times \mathbb{R} \rightarrow \mathbb{R}$ is a non-negative function, the above proposition ensures that every solution of problem $\left(S_{\lambda}^{f}\right)$ is either zero or positive.

Proof. Let $\theta \in \mathbb{Z}(1, m), \omega \in \mathbb{Z}(1, n)$ and

$$
\bar{x}(\theta, \omega)=\min \{\bar{x}(i, j): i \in \mathbb{Z}(1, m), j \in \mathbb{Z}(1, n)\} .
$$

If $\bar{x}(\theta, \omega)>0$, then it is clear that $\bar{x}(i, j)>0$ for all $i \in \mathbb{Z}(1, m), j \in \mathbb{Z}(1, n)$ and the proof is complete.

If $\bar{x}(\theta, \omega) \leq 0$, then $\bar{x}(\theta, \omega)=\min \{\bar{x}(i, j): i \in \mathbb{Z}(0, m+1), j \in \mathbb{Z}(0, n+1)\}$, since $\Delta_{1} \bar{x}(\theta-1, \omega)=$ $\bar{x}(\theta, \omega)-\bar{x}(\theta-1, \omega) \leq 0, \Delta_{2} \bar{x}(\theta, \omega-1)=\bar{x}(\theta, \omega)-\bar{x}(\theta, \omega-1) \leq 0$, and $\Delta_{1} \bar{x}(\theta, \omega)=\bar{x}(\theta+1, \omega)-$ $\bar{x}(\theta, \omega) \geq 0, \Delta_{2} \bar{x}(\theta, \omega)=\bar{x}(\theta, \omega+1)-\bar{x}(\theta, \omega) \geq 0, \phi_{p}(\eta)$ is increasing in $\eta$, and $\phi_{p}(0)=0$, we obtain

$$
\phi_{p}\left(\Delta_{1} \bar{x}(\theta, \omega)\right) \geq 0 \geq \phi_{p}\left(\Delta_{1} \bar{x}(\theta-1, \omega)\right) .
$$

Similarly,

$$
\phi_{p}\left(\Delta_{2} \bar{x}(\theta, \omega)\right) \geq 0 \geq \phi_{p}\left(\Delta_{2} \bar{x}(\theta, \omega-1)\right) .
$$

We get

$$
\Delta_{1}\left(\phi_{p}\left(\Delta_{1} \bar{x}(\theta-1, \omega)\right)\right)+\Delta_{2}\left(\phi_{p}\left(\Delta_{2} \bar{x}(\theta, \omega-1)\right)\right) \geq 0 .
$$

Thus, we have

$$
\phi_{p}\left(\Delta_{1} \bar{x}(\theta, \omega)\right)=\phi_{p}\left(\Delta_{1} \bar{x}(\theta-1, \omega)\right)=0 .
$$

That is $\bar{x}(\theta+1, \omega)=\bar{x}(\theta, \omega)=\bar{x}(\theta-1, \omega)$. If $\theta+1=m+1$, we get $\bar{x}(\theta, \omega)=0$. Otherwise, $(\theta+1) \in \mathbb{Z}(1, m)$. Replacing $\theta$ by $\theta+1$, we obtain $\bar{x}(\theta+2, \omega)=\bar{x}(\theta+1, \omega)$. Continuing this process $(m+1-\theta)$ times, we have $\bar{x}(\theta, \omega)=\bar{x}(\theta+1, \omega)=\bar{x}(\theta+2, \omega)=\cdots=\bar{x}(m+1, \omega)=0$. Similarly, 
we get $\bar{x}(\theta, \omega)=\bar{x}(\theta-1, \omega)=\bar{x}(\theta-2, \omega)=\cdots=\bar{x}(0, \omega)=0$. Therefore, $\bar{x}(i, \omega)=0$ for $i \in \mathbb{Z}(1, m)$. In the same way, we can show that $\bar{x} \equiv 0$ and the proof is complete.

\section{Main Results}

For each positive constant $h$, put

$$
\tau(h)=\frac{\sum_{j=1}^{n} \sum_{i=1}^{m} F((i, j), h)}{h^{p}} .
$$

Theorem 1. For every $(i, j) \in \mathbb{Z}(1, m) \times \mathbb{Z}(1, n)$, let $f((i, j), \cdot): \mathbb{R} \rightarrow \mathbb{R}$ be a non-negative continuous function. Assume that there exist three positive constants $b_{1}, b_{2}$ and $l$ with

$$
\frac{4 b_{1}}{(n+m)^{\frac{1}{p}} \cdot(m+n+2)^{\frac{p-1}{p}}}<l<\left(\frac{4}{n+m+2}\right)^{\frac{p-1}{p}} \cdot \frac{b_{2}}{(n+m)^{\frac{1}{p}}}
$$

such that

$\left(g_{1}\right) \max \left\{\tau\left(b_{1}\right), 2 \tau\left(b_{2}\right)\right\}<\frac{4^{p}}{3 n+3 m} \cdot \frac{\tau(l)}{(m+n+2)^{p-1}}$.

Then, for each $\lambda \in\left(\frac{3 n+3 m}{p \tau(l)}, \frac{4^{p}}{p(m+n+2)^{p-1} \cdot \max \left\{\tau\left(b_{1}\right), 2 \tau\left(b_{2}\right)\right\}}\right)$, problem $\left(S_{\lambda}^{f}\right)$ admits at least two positive solutions $x_{k}, k=1,2$.

Proof. Fix $\lambda$ as in the conclusion, and put $\Phi, \Psi, I_{\lambda}$ as defined in Section 2 for all $x \in S$. Let us employ Lemma 1 to our problem. Clearly, $\Phi$ and $\Psi$ satisfy assumptions $(A)$ and $(B)$ of Lemma 1 . Now, let $x_{1}$ and $x_{2}$ be two local minima for $I_{\lambda}$. Then $x_{1}$ and $x_{2}$ are critical points for $I_{\lambda}$, so, $x_{1}$ and $x_{2}$ are solutions of problem $\left(S_{\lambda}^{f}\right)$. Owing to Proposition 2 , one has $x_{1}(i, j) \geq 0$ and $x_{2}(i, j) \geq 0$ for all $(i, j) \in \mathbb{Z}(1, m) \times \mathbb{Z}(1, n)$. It follows that $t x_{1}(i, j)+(1-t) x_{2}(i, j) \geq 0$ for every $(i, j) \in \mathbb{Z}(1, m) \times \mathbb{Z}(1, n)$ and for every $0 \leq t \leq 1$. Hence, $\Psi\left(t x_{1}+(1-t) x_{2}\right) \geq 0$ for all $0 \leq t \leq 1$ and $(C)$ is verified. Moreover, put

$$
\rho_{1}=\frac{\left(4 b_{1}\right)^{p}}{p(m+n+2)^{p-1}} \quad \text { and } \quad \rho_{2}=\frac{\left(4 b_{2}\right)^{p}}{p(m+n+2)^{p-1}} .
$$

For all $x \in S$, we have

$$
\max _{\substack{i \in \mathbb{Z}(1, m) \\ j \in \mathbb{Z}(1, n)}}\{|x(i, j)|\} \leq \frac{(m+n+2)^{\frac{p-1}{p}}}{4} \cdot\|x\| .
$$

We obtain

$$
\max _{\substack{i \in \mathbb{Z}(1, m) \\ j \in \mathbb{Z}(1, n)}}\{|x(i, j)|\} \leq \frac{(m+n+2)^{\frac{p-1}{p}}}{4} \cdot\left(p \rho_{1}\right)^{\frac{1}{p}}=b_{1},
$$

for every $x \in S$ such that $\|x\| \leq\left(p \rho_{1}\right)^{\frac{1}{p}}$, and

$$
\max _{\substack{i \in \mathbb{Z}(1, m) \\ j \in \mathbb{Z}(1, n)}}\{|x(i, j)|\} \leq \frac{(m+n+2)^{\frac{p-1}{p}}}{4} \cdot\left(p \rho_{2}\right)^{\frac{1}{p}}=b_{2},
$$


for all $x \in S$ such that $\|x\| \leq\left(p \rho_{2}\right)^{\frac{1}{p}}$. It follows that

$$
\begin{aligned}
\sup _{\frac{x \in \Phi^{-1}\left(-\infty, \rho_{1}\right)}{\rho_{1}} \Psi(x)} & =\frac{\sup _{1} \sum_{j=1}^{n} \sum_{i=1}^{m} F((i, j), x(i, j))}{\rho_{1}} \\
& \leq \frac{\sum_{j=1}^{n} \sum_{i=1}^{m} F\left((i, j), b_{1}\right)}{\rho_{1}} \\
& =\frac{p(m+n+2)^{p-1}}{4^{p}} \tau\left(b_{1}\right),
\end{aligned}
$$

and

$$
\begin{aligned}
\sup _{\frac{x \in \Phi^{-1}\left(-\infty, \rho_{2}\right)}{\rho_{2}} \Psi(x)} & =\frac{\sup _{\sum_{j}} \sum_{j=1}^{n} \sum_{i=1}^{m} F((i, j), x(i, j))}{\rho_{2}} \\
& \leq \frac{\sum_{j=1}^{n} \sum_{i=1}^{m} F\left((i, j), b_{2}\right)}{\rho_{2}} \\
& =\frac{p\left(m+n+\rho^{p-1}\right.}{4^{p}} \tau\left(b_{2}\right) .
\end{aligned}
$$

Let $v \in S$ be defined by

$$
v(i, j)= \begin{cases}l, & \text { if }(i, j) \in \mathbb{Z}(1, m) \times \mathbb{Z}(1, n), \\ 0, & \text { if } i=0, j \in \mathbb{Z}(0, n+1) \text { or } i=m+1, j \in \mathbb{Z}(0, n+1), \\ 0, & \text { if } j=0, i \in \mathbb{Z}(0, m+1) \text { or } j=n+1, i \in \mathbb{Z}(0, m+1) .\end{cases}
$$

Clearly, we have $\Phi(v)=\frac{(2 n+2 m)}{p} \cdot l^{p}$. Hence, from $\frac{4 b_{1}}{(n+m)^{\frac{1}{p}}(m+n+2)^{\frac{p-1}{p}}}<l$, we get $2 \rho_{1}<\Phi(v)$ and from $l<\left(\frac{4}{n+m+2}\right)^{\frac{p-1}{p}} \cdot \frac{b_{2}}{(m+n)^{\frac{1}{p}}}$, we obtain $\Phi(v)<\frac{\rho_{2}}{2}$. Moreover,

$$
\frac{\Psi(v)}{\Phi(v)}=\frac{p \cdot \sum_{j=1}^{n} \sum_{i=1}^{m} F((i, j), l)}{(2 n+2 m) \cdot l^{p}}=\frac{p \tau(l)}{2 n+2 m}
$$

Therefore, owing to $\left(g_{1}\right)$, we can get assumptions $\left(a_{1}\right)$ and $\left(a_{2}\right)$ of Lemma 1 . Further, one has that

$$
\lambda \in\left(\frac{3 n+3 m}{p \tau(l)}, \frac{4^{p}}{p(m+n+2)^{p-1} \cdot \max \left\{\tau\left(b_{1}\right), 2 \tau\left(b_{2}\right)\right\}}\right) .
$$

Thus, we see from Lemma 1 that problem $\left(S_{\lambda}^{f}\right)$ admits at least two positive solutions $x_{k}$, $k=1,2$.

Remark 2. Clearly, problem $\left(E_{\lambda}^{f}\right)$ in [30] can be regarded as the special case $p=2$ of problem $\left(S_{\lambda}^{f}\right)$. In such a case, we get the set of $\lambda$ in Theorem 1 which is similar to ([30] Theorem 3.2) such that problem $\left(S_{\lambda}^{f}\right)$ admits at least two positive solutions. In Theorem 1, we get the set of $\lambda$ that is more specific than the set of $\lambda$ in ([30] Theorem 3.2), where $\lambda_{1}$ in the set of $\lambda$ is just known to be an eigenvalue but not given a definite expression. Compared with that, in this paper, the set of $\lambda$ we put forward can be calculated to any given number, so it is possible to get the full set of $\lambda$. Moreover, we obtain the existence of infinitely many solutions for problem $\left(S_{\lambda}^{f}\right)$ when $1<p<+\infty$, which extends the case of $p=2$, discussed in [30].

Now, we mark the discrete problem $\left(S_{\lambda}^{f}\right)$ as $\left(S_{\lambda}^{y, \beta}\right)$ when $f((i, j), x(i, j))=\beta(i, j) y(x(i, j))$, that is

$$
\Delta_{1}\left(\phi_{p}\left(\Delta_{1} x(i-1, j)\right)\right)+\Delta_{2}\left(\phi_{p}\left(\Delta_{2} x(i, j-1)\right)\right)+\lambda \beta(i, j) y(x(i, j))=0, \quad(i, j) \in \mathbb{Z}(1, m) \times \mathbb{Z}(1, n),
$$


with boundary conditions

$$
\begin{aligned}
& x(i, 0)=x(i, n+1)=0, \quad i \in \mathbb{Z}(0, m+1), \\
& x(0, j)=x(m+1, j)=0, \quad j \in \mathbb{Z}(0, n+1),
\end{aligned}
$$

where $\beta: \mathbb{Z}(1, m) \times \mathbb{Z}(1, n) \rightarrow \mathbb{R}$ is a non-negative and non-zero function and $y:[0,+\infty) \rightarrow \mathbb{R}$ is a continuous function such that $y(0)=0$.

Corollary 1. Assume that there exist three positive constants $b_{1}, b_{2}$ and $l$ with

$$
\frac{4 b_{1}}{(n+m)^{\frac{1}{p}} \cdot(m+n+2)^{\frac{p-1}{p}}}<l<\left(\frac{4}{n+m+2}\right)^{\frac{p-1}{p}} \cdot \frac{b_{2}}{(n+m)^{\frac{1}{p}}}
$$

such that

$$
\left(g_{2}\right) \max \left\{\frac{\int_{0}^{b_{1}} y(\xi) d \xi}{b_{1}^{p}}, \frac{2 \int_{0}^{b_{2}} y(\xi) d \xi}{b_{2}^{p}}\right\}<\frac{4^{p} \int_{0}^{l} y(\xi) d \xi}{(3 n+3 m)^{p} \cdot(m+n+2)^{p-1}}
$$

Then, for every

$$
\begin{aligned}
& \lambda \in\left(\frac{(3 n+3 m) \cdot l^{p}}{p \cdot \sum_{j=1}^{n} \sum_{i=1}^{m} \int_{0}^{l} \beta(i, j) y(\xi) d \xi},\right. \\
& \frac{4^{p}}{p(m+n+2)^{p-1} \cdot \max \left\{\frac{\sum_{j=1}^{n} \sum_{i=1}^{m} \int_{0}^{b_{1}} \beta(i, j) y(\xi) d \xi}{b_{1}^{p}}, \frac{2 \sum_{j=1}^{n} \sum_{i=1}^{m} \int_{0}^{b_{2}} \beta(i, j) y(\xi) d \xi}{b_{2}^{p}}\right\}},
\end{aligned}
$$

problem $\left(S_{\lambda}^{y, \beta}\right)$ admits at least two positive solutions.

Proof. Put

$$
f((i, j), r)= \begin{cases}\beta(i, j) y(r), & \text { if } r \geq 0, \\ 0, & \text { if } r<0,\end{cases}
$$

for all $(i, j) \in \mathbb{Z}(1, m) \times \mathbb{Z}(1, n)$ and $r \in \mathbb{R}$. By Theorem 1 and $\left(g_{2}\right)$, we obtain the conclusion.

Theorem 2. For every $(i, j) \in \mathbb{Z}(1, m) \times \mathbb{Z}(1, n)$, let $f((i, j), \cdot): \mathbb{R} \rightarrow \mathbb{R}$ be a non-negative continuous function. Assume that there exist three positive constants $b_{1}, b_{2}$, and $l$ with

$$
\frac{4 b_{1}}{(m+n+2)^{\frac{p-1}{p}}} \cdot\left(\frac{1}{2 n+2 m}\right)^{\frac{1}{p}}<l<\frac{b_{2}}{(n+m)^{\frac{1}{p}}} \cdot\left(\frac{4}{m+n+2}\right)^{\frac{p-1}{p}}
$$

such that

$\left(g_{3}\right) \max \left\{\tau\left(b_{1}\right), 2 \tau\left(b_{2}\right)\right\}<\left(\frac{4}{n+m+2}\right)^{p-1} \cdot \frac{\tau(l)}{(n+m)}$.

Then, for all $\lambda \in\left(\frac{4 n+4 m}{p \tau(l)}, \frac{4^{p}}{p(m+n+2)^{p-1} \cdot \max \left\{\tau\left(b_{1}\right), 2 \tau\left(b_{2}\right)\right\}}\right)$, problem $\left(S_{\lambda}^{f}\right)$ admits at least two positive solutions. 
Proof. Let

$$
\rho_{1}=\frac{\left(4 b_{1}\right)^{p}}{p(m+n+2)^{p-1}} \quad \text { and } \quad \rho_{2}=\frac{\left(4 b_{2}\right)^{p}}{p(m+n+2)^{p-1}}
$$

We have

$$
\frac{\sup _{x \in \Phi^{-1}\left(-\infty, \rho_{1}\right)} \Psi(x)}{\rho_{1}} \leq \frac{p(m+n+2)^{p-1}}{4^{p}} \cdot \tau\left(b_{1}\right)
$$

and

$$
\frac{\sup _{x \in \Phi^{-1}\left(-\infty, \rho_{2}\right)} \Psi(x)}{\rho_{2}} \leq \frac{p(m+n+2)^{p-1}}{4^{p}} \cdot \tau\left(b_{2}\right)
$$

For $v \in S$ defined as

$$
v(i, j)= \begin{cases}l, & \text { if }(i, j) \in \mathbb{Z}(1, m) \times \mathbb{Z}(1, n), \\ 0, & \text { if } i=0, j \in \mathbb{Z}(0, n+1) \text { or } i=m+1, j \in \mathbb{Z}(0, n+1), \\ 0, & \text { if } j=0, i \in \mathbb{Z}(0, m+1) \text { or } j=n+1, i \in \mathbb{Z}(0, m+1),\end{cases}
$$

we obtain $\Phi(v)=\frac{(2 n+2 m)}{p} \cdot l^{p}$. From $\frac{4 b_{1}}{(m+n+2)^{\frac{p-1}{p}}} \cdot\left(\frac{1}{2 n+2 m}\right)^{\frac{1}{p}}<l$, we have $\rho_{1}<\Phi(v)$ and from $l<\frac{b_{2}}{(n+m)^{\frac{1}{p}}} \cdot\left(\frac{4}{m+n+2}\right)^{\frac{p-1}{p}}$, we get $\Phi(v)<\frac{\rho_{2}}{2}$. It is clear that,

$$
\frac{\Psi(v)}{\Phi(v)}=\frac{p \tau(l)}{2 n+2 m}
$$

Owing to $\left(g_{3}\right)$, problem $\left(S_{\lambda}^{f}\right)$ admits at least two positive solutions.

Theorem 3. For every $(i, j) \in \mathbb{Z}(1, m) \times \mathbb{Z}(1, n)$, let $f((i, j), \cdot): \mathbb{R} \rightarrow \mathbb{R}$ be a non-negative continuous function. Assume that there are positive constants $e_{1}, e_{2}, e_{3}$ and $d$ with

$$
\frac{4 e_{1}}{(m+n+2)^{\frac{p-1}{p}}} \cdot\left(\frac{1}{2 n+2 m}\right)^{\frac{1}{p}}<d<\frac{4 e_{2}}{(m+n+2)^{\frac{p-1}{p}}} \cdot\left(\frac{1}{2 n+2 m}\right)^{\frac{1}{p}} \text { and } e_{2}<e_{3}
$$

such that

$\left(g_{4}\right) \max \left\{\tau\left(e_{1}\right), \tau\left(e_{2}\right), \frac{\sum_{j=1}^{n} \sum_{i=1}^{m} F\left((i, j), e_{3}\right)}{e_{3}^{p}-e_{2}^{p}}\right\}<\frac{4^{p-1} \tau(d)}{(n+m) \cdot(m+n+2)^{p-1}}$.

Then, for all

$$
\begin{aligned}
\lambda \in\left(\frac{(2 n+2 m) \cdot 4^{p}}{4^{p} \cdot \tau(d) p-(2 n+2 m) \cdot p \tau\left(e_{1}\right) \cdot(m+n+2)^{p-1}}\right. \\
\left.\frac{4^{p}}{p \cdot(m+n+2)^{p-1} \max \left\{\tau\left(e_{1}\right), \tau\left(e_{2}\right), \frac{\sum_{j=1}^{n} \sum_{i=1}^{m} F\left((i, j), e_{3}\right)}{e_{3}^{p}-e_{2}^{p}}\right\}}\right),
\end{aligned}
$$

problem $\left(S_{\lambda}^{f}\right)$ admits at least two positive solutions $x_{k}, k=1,2$.

Proof. Let

$$
t_{1}=\frac{\left(4 e_{1}\right)^{p}}{p(m+n+2)^{p-1}}
$$




$$
\begin{aligned}
& t_{2}=\frac{\left(4 e_{2}\right)^{p}}{p(m+n+2)^{p-1}}, \\
& t_{3}=\frac{\left(4 e_{3}\right)^{p}-\left(4 e_{2}\right)^{p}}{p(m+n+2)^{p-1}} .
\end{aligned}
$$

By (1), we have

$$
\max _{\substack{i \in \mathbb{Z}(1, m) \\ j \in \mathbb{Z}(1, n)}}\{|x(i, j)|\} \leq \frac{(m+n+2)^{\frac{p-1}{p}}}{4} \cdot\left(p t_{1}\right)^{\frac{1}{p}}=e_{1},
$$

for all $x \in S$ such that $\|x\| \leq\left(p t_{1}\right)^{\frac{1}{p}}$, and

$$
\max _{\substack{i \in \mathbb{Z}(1, m) \\ j \in \mathbb{Z}(1, n)}}\{|x(i, j)|\} \leq \frac{(m+n+2)^{\frac{p-1}{p}}}{4} \cdot\left(p t_{2}\right)^{\frac{1}{p}}=e_{2},
$$

for each $x \in S$ such that $\|x\| \leq\left(p t_{2}\right)^{\frac{1}{p}}$, and

$$
\max _{\substack{i \in \mathbb{Z}(1, m) \\ j \in \mathbb{Z}(1, n)}}\{|x(i, j)|\} \leq \frac{(m+n+2)^{\frac{p-1}{p}}}{4} \cdot\left(p t_{2}+p t_{3}\right)^{\frac{1}{p}}=e_{3},
$$

for all $x \in S$ such that $\|x\| \leq\left(p t_{2}+p t_{3}\right)^{\frac{1}{p}}$. One has

$$
\begin{aligned}
& \varphi\left(t_{1}\right)=\inf _{x \in \Phi^{-1}\left(-\infty, t_{1}\right)} \frac{\left(\sup _{\Psi \in \Phi^{-1}\left(-\infty, t_{1}\right)} \Psi(x)\right)-\Psi(x)}{t_{1}-\Phi(x)} \\
& \leq \frac{\sup _{x \in \Phi^{-1}\left(-\infty, t_{1}\right)} \Psi(x)}{t_{1}} \\
& \sup \sum_{j=1}^{n} \sum_{i=1}^{m} F((i, j), x(i, j))
\end{aligned}
$$

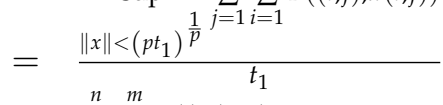

$$
\begin{aligned}
& \leq \frac{\sum_{j=1}^{n} \sum_{i=1}^{m} F\left((i, j), e_{1}\right)}{t_{1}} \\
& =\frac{p \tau\left(e_{1}\right) \cdot(m+n+2)^{p-1}}{4^{p}} \text {, } \\
& \varphi\left(t_{2}\right)=\inf _{\substack{x \in \Phi^{-1}\left(-\infty, t_{2}\right) \\
\sup }} \frac{\left(\sup _{x \in \Phi^{-1}(-\infty)} \Psi(x)\right)-\Psi(x)}{t_{2}-\Phi(x)} \\
& \leq \frac{\sup _{x \in \Phi^{-1}\left(-\infty, t_{2}\right)} \Psi(x)}{t_{2}} \\
& =\frac{\|x\|<\left(p t_{2}\right)^{\frac{1}{p}} \sum_{j=1}^{n} \sum_{i=1} F((i, j), x(i, j))}{t_{2}} \\
& \leq \frac{\sum_{j=1}^{n} \sum_{i=1}^{m} F\left((i, j), e_{2}\right)}{t_{2}} \\
& =\frac{p \tau\left(e_{2}\right) \cdot(m+n+2)^{p-1}}{4^{p}},
\end{aligned}
$$


and

$$
\begin{aligned}
& \gamma\left(t_{2}, t_{3}\right)=\frac{\sup _{x \in \Phi^{-1}\left(-\infty, t_{2}+t_{3}\right)} \Psi(x)}{\operatorname{ts}_{3} \Psi(x)} \\
& =\frac{\|x\|<\left(p t_{2}+p t_{3}\right)^{\frac{1}{p}}}{t_{3}} \\
& =\frac{t_{3} \sum_{j=1}^{n} \sum_{i=1}^{m} F((i, j), x(i, j))}{\sup } \\
& =\frac{\|x\|<\left(p t_{2}+p t_{3}\right)^{\frac{1}{p^{p}}} \sum^{j=1} \sum_{i=1}((i, j),(i, j))}{t_{3} m} \\
& \leq \frac{\sum_{j=1}^{n} \sum_{i=1}^{m} F\left((i, j), e_{3}\right)}{\sum_{j=1}^{n} t_{3} t_{i} F\left((i, j), e_{3}\right) \cdot(m b} \\
& =\frac{p \sum_{j=1}^{n} \sum_{i=1}^{m} F\left((i, j), e_{3}\right) \cdot(m+n+2)^{p-1}}{\left(4 e_{3}\right)^{p}-\left(4 e_{2}\right)^{p}} .
\end{aligned}
$$

Let $u \in S$ be defined by

$$
u(i, j)= \begin{cases}d, & \text { if }(i, j) \in \mathbb{Z}(1, m) \times \mathbb{Z}(1, n), \\ 0, & \text { if } i=0, j \in \mathbb{Z}(0, n+1) \text { or } i=m+1, j \in \mathbb{Z}(0, n+1), \\ 0, & \text { if } j=0, i \in \mathbb{Z}(0, m+1) \text { or } j=n+1, i \in \mathbb{Z}(0, m+1) .\end{cases}
$$

Then we have

$$
\begin{aligned}
\beta\left(t_{1}, t_{2}\right) & =\inf _{x \in \Phi^{-1}\left(-\infty, t_{1}\right)} \sup _{y \in \Phi^{-1}\left[t_{1}, t_{2}\right)} \frac{\Psi(y)-\Psi(x)}{\Phi(y)-\Phi(x)} \\
& \geq \inf _{x \in \Phi^{-1}\left(-\infty, t_{1}\right)} \frac{\Psi(u)-\Psi(x)}{\Phi(u)-\Phi(x)} \\
& \geq \inf _{x \in \Phi^{-1}\left(-\infty, t_{1}\right)} \frac{\Psi(u)-\Psi(x)}{\Phi(u)} \\
& \geq \frac{\Psi(u)}{\Phi(u)}-\frac{x \in \Phi^{-1}\left(-\infty, t_{1}\right)}{t_{1}} \\
& =\frac{\sum_{j=1}^{n} \sum_{i=1}^{m} F((i, j), d)}{\frac{(2 n+2 m) \cdot d p}{p}}-\frac{\|x\|<\left(p t_{1}\right)^{\frac{1}{p}} \sum_{j=1}^{n} \sum_{i=1}^{m} F((i, j), x(i, j))}{t_{1}} \\
& \geq \frac{p \sum_{j=1}^{n} \sum_{i=1}^{m} F((i, j), d)}{(2 n+2 m) d^{p}}-\frac{\sum_{j=1}^{n} \sum_{i=1}^{m} F\left((i, j), e_{1}\right)}{t_{1}} \\
& =\frac{p \tau(d)}{2 n+2 m}-\frac{p \tau\left(e_{1}\right) \cdot(m+n+2)^{p-1}}{4^{p}} .
\end{aligned}
$$

Hence, from $\frac{4 e_{1}}{(m+n+2)^{\frac{p-1}{p}}} \cdot\left(\frac{1}{2 n+2 m}\right)^{\frac{1}{p}}<d<\frac{4 e_{2}}{(m+n+2)^{\frac{p-1}{p}}} \cdot\left(\frac{1}{2 n+2 m}\right)^{\frac{1}{p}}, e_{2}<e_{3}$ and $\left(g_{4}\right)$, we obtain $\varphi\left(t_{1}\right)<\beta\left(t_{1}, t_{2}\right), \varphi\left(t_{2}\right)<\beta\left(t_{1}, t_{2}\right)$ and $\gamma\left(t_{2}, t_{3}\right)<\beta\left(t_{1}, t_{2}\right)$. Then it is clear that for all

$$
\begin{aligned}
\lambda \in\left(\frac{(2 n+2 m) \cdot 4^{p}}{4^{p} \cdot \tau(d) p-(2 n+2 m) \cdot p \tau\left(e_{1}\right) \cdot(m+n+2)^{p-1}},\right. \\
\left.\frac{4^{p}}{p \cdot(m+n+2)^{p-1} \max \left\{\tau\left(e_{1}\right), \tau\left(e_{2}\right), \frac{\sum_{j=1}^{n} \sum_{i=1}^{m} F\left((i, j), e_{3}\right)}{e_{3}^{p}-e_{2}^{p}}\right\}}\right),
\end{aligned}
$$

problem $\left(S_{\lambda}^{f}\right)$ admits at least two positive solutions $x_{k}, k=1,2$. 
Let

$$
B^{\infty}=\limsup _{x \rightarrow+\infty}\left(\frac{\sum_{j=1}^{n} \sum_{i=1}^{m} F((i, j), x)}{x^{p}}\right) .
$$

When $B^{\infty}=+\infty$, we agree to read $\frac{1}{B^{\infty}}=0$.

Theorem 4. Assume that there exist two real sequences $\left\{\alpha_{t}\right\}$ and $\left\{\beta_{t}\right\}$, with $\lim _{t \rightarrow+\infty} \beta_{t}=+\infty$, such that

$$
0 \leq \alpha_{t}<\frac{4 \beta_{t}}{(2 n+2 m)^{\frac{1}{p}} \cdot(m+n+2)^{\frac{p-1}{p}}}, \text { for all } t \in \mathbb{N},
$$

and

$$
Q_{\infty}=\lim _{t \rightarrow+\infty} \frac{\sum_{j=1}^{n} \sum_{i=1}^{m} \max _{|x| \leq \beta_{t}} F((i, j), x)-\sum_{j=1}^{n} \sum_{i=1}^{m} F\left((i, j), \alpha_{t}\right)}{\left(4 \beta_{t}\right)^{p}-(2 n+2 m)\left(\alpha_{t}\right)^{p}(m+n+2)^{p-1}}<\frac{B^{\infty}}{(2 n+2 m) \cdot(m+n+2)^{p-1}} .
$$

Then, for every $\lambda \in\left(\frac{2 n+2 m}{p} \cdot \frac{1}{B^{\infty}}, \frac{1}{p(m+n+2)^{p-1}} \cdot \frac{1}{Q_{\infty}}\right)$, problem $\left(S_{\lambda}^{f}\right)$ admits an unbounded sequence of solutions.

Proof. Fix $\lambda \in\left(\frac{2 n+2 m}{p} \cdot \frac{1}{B^{\infty}}, \frac{1}{p(m+n+2)^{p-1}} \cdot \frac{1}{Q_{\infty}}\right)$ and put

$$
\gamma_{t}=\frac{\left(4 \beta_{t}\right)^{p}}{p(m+n+2)^{p-1}}, \quad \text { for each } t \in \mathbb{N} .
$$

From (1), we have

$$
\max _{\substack{i \in \mathbb{Z}(1, m) \\ j \in \mathbb{Z}(1, n)}}\{|x(i, j)|\} \leq \frac{(m+n+2)^{\frac{p-1}{p}}}{4} \cdot\left(p \gamma_{t}\right)^{\frac{1}{p}}=\beta_{t}, \text { for every } x \in S,
$$

such that $\|x\| \leq\left(p \gamma_{t}\right)^{\frac{1}{p}}$ for each $t \in \mathbb{N}$, and we obtain

$$
\varphi\left(\gamma_{t}\right) \leq \inf _{x \in \Phi^{-1}\left(-\infty, \gamma_{t}\right)} \frac{\sum_{j=1}^{n} \sum_{i=1}^{m} \max _{x \mid \leq \beta_{t}} F((i, j), x)-\sum_{j=1}^{n} \sum_{i=1}^{m} F((i, j), x)}{\frac{\left(4 \beta_{t}\right)^{p}}{p(m+n+2)^{p-1}}-\frac{\|x\|^{p}}{p}} .
$$

Now, we choose $\eta_{t} \in S$, defined by

$$
\eta_{t}(i, j)= \begin{cases}\alpha_{t}, & \text { if }(i, j) \in \mathbb{Z}(1, m) \times \mathbb{Z}(1, n), \\ 0, & \text { if } i=0, j \in \mathbb{Z}(0, n+1) \text { or } i=m+1, j \in \mathbb{Z}(0, n+1), \\ 0, & \text { if } j=0, i \in \mathbb{Z}(0, m+1) \text { or } j=n+1, i \in \mathbb{Z}(0, m+1) .\end{cases}
$$

Clearly, we get $\Phi\left(\eta_{t}\right)=\left(\frac{2 n+2 m}{p}\right) \alpha_{t}^{p}$ and $\left\|\eta_{t}\right\|^{p}<p \gamma_{t}$. One has

$$
\varphi\left(\gamma_{t}\right) \leq \frac{\left(\sum_{j=1}^{n} \sum_{i=1}^{m} \max _{|x| \leq \beta_{t}} F((i, j), x)-\sum_{j=1}^{n} \sum_{i=1}^{m} F\left((i, j), \alpha_{t}\right)\right) \cdot p(m+n+2)^{p-1}}{\left(4 \beta_{t}\right)^{p}-(2 n+2 m) \alpha_{t}^{p} \cdot(m+n+2)^{p-1}} .
$$


Therefore,

$$
\gamma \leq \lim _{t \rightarrow+\infty} \varphi\left(\gamma_{t}\right) \leq p Q_{\infty} \cdot(m+n+2)^{p-1}<+\infty .
$$

Now, we need to prove that $I_{\lambda}$ is unbounded from below. To this end, we first assume that $B^{\infty}=$ $+\infty$. Fix $M$ such that $M>\frac{2 n+2 m}{p \lambda}$ and let $\left\{e_{t}\right\}$ be a sequence of positive numbers, with $\lim _{t \rightarrow+\infty} e_{t}=+\infty$, such that $\sum_{j=1}^{n} \sum_{i=1}^{m} F\left((i, j), e_{t}\right)>M e_{t}^{p}, \forall t \in \mathbb{N}$. Define a sequence $\left\{d_{t}\right\}$ in $S$ with

$$
d_{t}(i, j)= \begin{cases}e_{t}, & \text { if }(i, j) \in \mathbb{Z}(1, m) \times \mathbb{Z}(1, n), \\ 0, & \text { if } i=0, j \in \mathbb{Z}(0, n+1) \text { or } i=m+1, j \in \mathbb{Z}(0, n+1), \\ 0, & \text { if } j=0, i \in \mathbb{Z}(0, m+1) \text { or } j=n+1, i \in \mathbb{Z}(0, m+1) .\end{cases}
$$

It is clear that

$$
I_{\lambda}\left(d_{t}\right)=\left(\frac{2 n+2 m}{p}\right) e_{t}^{p}-\lambda \sum_{j=1}^{n} \sum_{i=1}^{m} F\left((i, j), e_{t}\right)<\left(\frac{2 n+2 m}{p}\right) e_{t}^{p}-\lambda M e_{t}^{p},
$$

which implies that $\lim _{t \rightarrow+\infty} I_{\lambda}\left(d_{t}\right)=-\infty$. Next, we assume that $B^{\infty}<+\infty$ and take $\varepsilon>0$ such that $\varepsilon<B^{\infty}-\frac{2 n+2 m}{p \lambda}$. Then we have the sequence of positive numbers $\left\{e_{t}\right\}$ such that $\lim _{t \rightarrow+\infty} e_{t}=+\infty$ and

$$
\left(B^{\infty}-\varepsilon\right) e_{t}^{p}<\sum_{j=1}^{n} \sum_{i=1}^{m} F\left((i, j), e_{t}\right)<\left(B^{\infty}+\varepsilon\right) e_{t}^{p}, \quad \forall t \in \mathbb{N} .
$$

It is easy to see that

$$
I_{\lambda}\left(d_{t}\right)<\left(\frac{2 n+2 m}{p}-\lambda B^{\infty}+\lambda \varepsilon\right) \cdot e_{t}^{p} .
$$

So, $\lim _{t \rightarrow+\infty} I_{\lambda}\left(d_{t}\right)=-\infty$ and $I_{\lambda}$ is unbounded from below. The proof is complete.

Remark 3. When $B^{\infty}=+\infty$, according to Theorem 4 , we have that for every $\lambda \in\left(0, \frac{1}{p(m+n+2)^{p-1}} \cdot \frac{1}{Q_{\infty}}\right)$, problem $\left(S_{\lambda}^{f}\right)$ admits an unbounded sequence of solutions.

Set

$$
D_{\infty}=\liminf _{x \rightarrow+\infty} \frac{\sum_{j=1}^{n} \sum_{i=1}^{m} \max _{|\xi| \leq x} F((i, j), \xi)}{x^{p}} .
$$

When $D_{\infty}=0$, we agree to read $\frac{1}{D_{\infty}}=+\infty$.

Theorem 5. Assume that $D_{\infty}<\frac{4^{p} B^{\infty}}{(2 n+2 m)(m+n+2)^{p-1}}$. Then, for all

$$
\lambda \in\left(\frac{2 n+2 m}{p} \cdot \frac{1}{B^{\infty}}, \frac{4^{p}}{p(m+n+2)^{p-1}} \cdot \frac{1}{D_{\infty}}\right)
$$

problem $\left(S_{\lambda}^{f}\right)$ admits an unbounded sequence of solutions.

Proof. Let $\left\{\beta_{t}\right\}$ be a sequence of positive numbers with $\lim _{t \rightarrow+\infty} \beta_{t}=+\infty$ and

$$
\lim _{t \rightarrow+\infty} \frac{\sum_{j=1}^{n} \sum_{i=1}^{m} \max _{|x| \leq \beta_{t}} F((i, j), x)}{\left(\beta_{t}\right)^{p}}=D_{\infty} .
$$


Setting $\alpha_{t}=0$ for each $t \in \mathbb{N}$, we can reach the conclusion by Theorem 4 .

Remark 4. When $B^{\infty}=+\infty$, according to the Theorem 5, we have that for every $\lambda \in\left(0, \frac{4^{p}}{p(m+n+2)^{p-1}} \cdot \frac{1}{D_{\infty}}\right)$, problem $\left(S_{\lambda}^{f}\right)$ admits an unbounded sequence of solutions. When $D_{\infty}=0$, according to the Theorem 5 , we have that for every $\lambda \in\left(\frac{2 n+2 m}{p} \cdot \frac{1}{B^{\infty}},+\infty\right)$, problem $\left(S_{\lambda}^{f}\right)$ admits an unbounded sequence of solutions. When $B^{\infty}=+\infty$ and $D_{\infty}=0$, according to the Theorem 5 , we have that for every $\lambda>0$, problem $\left(S_{\lambda}^{f}\right)$ admits an unbounded sequence of solutions.

Now, we mark the problem $\left(S_{\lambda}^{f}\right)$ as $\left(S_{\lambda}^{g, \alpha}\right)$ when $f((i, j), x(i, j))=\alpha(i, j) g(x(i, j))$, that is

$$
\Delta_{1}\left(\phi_{p}\left(\Delta_{1} x(i-1, j)\right)\right)+\Delta_{2}\left(\phi_{p}\left(\Delta_{2} x(i, j-1)\right)\right)+\lambda \alpha(i, j) g(x(i, j))=0, \quad(i, j) \in \mathbb{Z}(1, m) \times \mathbb{Z}(1, n),
$$

with boundary conditions

$$
\begin{aligned}
& x(i, 0)=x(i, n+1)=0, \quad i \in \mathbb{Z}(0, m+1), \\
& x(0, j)=x(m+1, j)=0, \quad j \in \mathbb{Z}(0, n+1),
\end{aligned}
$$

where $\alpha: \mathbb{Z}(1, m) \times \mathbb{Z}(1, n) \rightarrow \mathbb{R}$ is a non-negative and non-zero function and $g:[0,+\infty) \rightarrow \mathbb{R}$ is a non-negative continuous function such that $g(0)=0$.

Corollary 2. Assume that

$$
\liminf _{x \rightarrow+\infty} \frac{\int_{0}^{x} g(s) d s}{x^{p}}<\frac{4^{p}}{(2 n+2 m)(m+n+2)^{p-1}} \limsup _{x \rightarrow+\infty}\left(\frac{\int_{0}^{x} g(s) d s}{x^{p}}\right) .
$$

Then, for every

$$
\lambda \in\left(\frac{2 n+2 m}{p\left(\limsup _{x \rightarrow+\infty}\left(\frac{\sum_{j=1}^{n} \sum_{i=1}^{m} \int_{0}^{x} \alpha(i, j) g(s) d s}{x^{p}}\right)\right)^{-}},\right.
$$

problem $\left(S_{\lambda}^{g, \alpha}\right)$ admits an unbounded sequence of positive solutions.

Proof. Set

$$
f((i, j), x)= \begin{cases}\alpha(i, j) g(x), & x \geq 0 \\ 0, & x<0\end{cases}
$$


for each $(i, j) \in \mathbb{Z}(1, m) \times \mathbb{Z}(1, n)$ and $x \in \mathbb{R}$. Taking Proposition 2 into account, our goal can be obtained owing to Theorem 5 .

\section{Examples}

We give two examples to illustrate our results.

Example 1. Put $p=3, b_{1}=1, b_{2}=14, l=2, m=2$ and $n=3$. Let $\beta: \mathbb{Z}(1,2) \times \mathbb{Z}(1,3) \rightarrow \mathbb{R}$ be $a$ positive function and set $A=\sum_{j=1}^{3} \sum_{i=1}^{2} \beta(i, j)$. Make $y:[0,+\infty) \rightarrow \mathbb{R}$ be the function defined as follows

$$
y(r)= \begin{cases}r^{14}, & 0 \leq r<2, \\ (4-r)^{14}, & 2 \leq r<3 \\ 1, & 3 \leq r .\end{cases}
$$

One has

$$
\begin{gathered}
\int_{0}^{1} y(\xi) d \xi=\int_{0}^{1} r^{14} d r=\frac{1}{15} \\
\frac{2}{14^{3}} \cdot \int_{0}^{14} y(\xi) d \xi=\frac{2}{14^{3}} \cdot\left[\int_{0}^{2} r^{14} d r+\int_{2}^{3}(4-r)^{14} d r+\int_{3}^{14} 1 d r\right]=\frac{1095}{343}
\end{gathered}
$$

and

$\frac{4^{3}}{(3 \times 2+3 \times 3)\left(2^{3} \times(3+2+2)^{2}\right)} \int_{0}^{2} y(\xi) d \xi=\frac{4^{3}}{(3 \times 2+3 \times 3)\left(2^{3} \times(3+2+2)^{2}\right)} \int_{0}^{2} r^{14} d r=\frac{262,144}{11,025}$.

We get

$$
\max \left\{\int_{0}^{1} y(\xi) d \xi, \frac{2}{14^{3}} \cdot \int_{0}^{14} y(\xi) d \xi\right\}<\frac{4^{3}}{(3 \times 2+3 \times 3) \times\left(2^{3} \times(3+2+2)^{2}\right)} \times \int_{0}^{2} y(\xi) d \xi,
$$

and

$$
\frac{4 \times 1}{(2+3)^{\frac{1}{3}} \cdot(2+3+2)^{\frac{2}{3}}}<2<\left(\frac{4}{2+3+2}\right)^{\frac{2}{3}} \times \frac{14}{(2+3)^{\frac{1}{3}}} .
$$

Applying to Corollary 1, for each $\lambda \in\left(\frac{75}{4096 \mathrm{~A}}, \frac{448}{3285 \mathrm{~A}}\right)$, the following problem

$$
\Delta_{1}\left(\phi_{3}\left(\Delta_{1} x(i-1, j)\right)\right)+\Delta_{2}\left(\phi_{3}\left(\Delta_{2} x(i, j-1)\right)\right)+\lambda \beta(i, j) y(x(i, j))=0, \quad(i, j) \in \mathbb{Z}(1,2) \times \mathbb{Z}(1,3),
$$

with boundary conditions

$$
\begin{aligned}
& x(i, 0)=x(i, 4)=0, \quad i \in \mathbb{Z}(0,3), \\
& x(0, j)=x(3, j)=0, \quad j \in \mathbb{Z}(0,4),
\end{aligned}
$$

admits at least two positive solutions.

Example 2. Put $m=2, n=2$ and $p=2$. Set $\alpha(i, j)=1$, for $(i, j) \in \mathbb{Z}(1,2) \times \mathbb{Z}(1,2)$ and let $g:[0,+\infty) \rightarrow \mathbb{R}$ be the function defined as follows

$$
g(x)= \begin{cases}\frac{18}{5} x+x \cos (\ln (x))+2 x \sin (\ln (x)), & x>0 \\ 0, & x=0 .\end{cases}
$$

We have

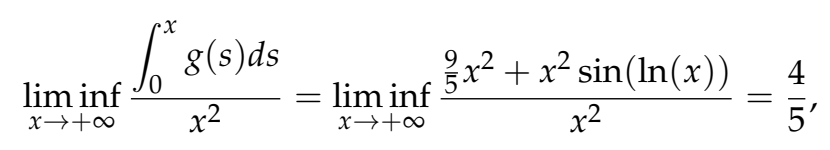


and

$$
\limsup _{x \rightarrow+\infty} \frac{\int_{0}^{x} g(s) d s}{x^{2}}=\limsup _{x \rightarrow+\infty} \frac{\frac{9}{5} x^{2}+x^{2} \sin (\ln (x))}{x^{2}}=\frac{14}{5} .
$$

Since

$$
\frac{4^{2}}{(2 \times 2+2 \times 2) \times(2+2+2)^{2-1}}>\frac{\frac{4}{5}}{\frac{14}{5}}
$$

one has

$$
\liminf _{x \rightarrow+\infty} \frac{\int_{0}^{x} g(s) d s}{x^{2}}<\frac{4^{2}}{(2 \times 2+2 \times 2) \times(2+2+2)^{2-1}} \cdot \limsup _{x \rightarrow+\infty} \frac{\int_{0}^{x} g(s) d s}{x^{2}} .
$$

By Corollary 2, for each $\lambda \in\left(\frac{5}{14}, \frac{5}{12}\right)$, the following problem

$$
\begin{array}{ll} 
& \Delta_{1}\left(\phi_{2}\left(\Delta_{1} x(i-1, j)\right)\right)+\Delta_{2}\left(\phi_{2}\left(\Delta_{2} x(i, j-1)\right)\right) \\
+\quad & \lambda\left[\frac{18}{5} x(i, j)+x(i, j) \cos (\ln (x(i, j)))+2 x(i, j) \sin (\ln (x(i, j)))\right]=0,
\end{array}
$$

for $(i, j) \in \mathbb{Z}(1,2) \times \mathbb{Z}(1,2)$, with boundary conditions

$$
\begin{aligned}
& x(i, 0)=x(i, 3)=0, \quad i \in \mathbb{Z}(0,3), \\
& x(0, j)=x(3, j)=0, \quad j \in \mathbb{Z}(0,3),
\end{aligned}
$$

admits an unbounded sequence of positive solutions.

\section{Conclusions}

In this paper, we consider the existence of multiple solutions for a partial discrete Dirichlet problem $\left(S_{\lambda}^{f}\right)$ involving the $p$-Laplacian. For problem $\left(S_{\lambda}^{f}\right)$, in the framework of variational methods, we give that the set of $\lambda$ can be more specific than that in the special case of $p=2$ ([30] Theorem 3.2). Furthermore, with appropriate assumptions on the nonlinear term, we get that problem $\left(S_{\lambda}^{f}\right)$ admits an unbounded sequence of solutions by using Theorem 2.1 of [34]. By the method of the critical point theory, we obtain sufficient conditions to guarantee the existence of multiple solutions for problem $\left(S_{\lambda}^{f}\right)$, which are different from these conditions in $[30,33,34]$. In the future, we will consider problem $\left(S_{\lambda}^{f}\right)$ with fewer and looser constraints and we could consider the existence of partial difference equations with different boundary value conditions.

Author Contributions: All authors contributed equally and significantly in writing this paper. All authors have read and agreed to the published version of the manuscript.

Funding: This work is supported by the National Natural Science Foundation of China (Grant No. 11971126), the Program for Changiiang Scholars and Innovative Research Team in University (Grant No. IRT_16R16), the Innovation Research for the postgraduates of Guangzhou University (Grant No. 2019GDJC-D04), and Science and Technology Planning Project of Guangdong Province of China (Grant No. 2020A1414010106).

Conflicts of Interest: The authors declare that they have no conflict of interest.

\section{References}

1. Elaydi, S. An Introduction to Difference Equations, 3rd ed.; Springer: New York, NY, USA, 2011.

2. Kelly, W.G.; Peterson, A.C. Difference Equations: An Introduction with Applications; Academic Press: San Diego, CA, USA; New York, NY, USA; Basel, Switzerland, 1991.

3. Yu, J.S.; Zheng, B. Modeling Wolbachia infection in mosquito population via discrete dynamical model. J. Differ. Equ. Appl. 2019, 25, 1549-1567. [CrossRef]

4. Balanov, Z.; Garcia-Azpeitia, C.; Krawcewicz, W. On variational and topological methods in nonlinear difference equations. Commun. Pure Appl. Anal. 2018, 17, 2813-2844. [CrossRef] 
5. Zhou, Z.; Yu, J.S.; Chen, Y.M. Homoclinic solutions in periodic difference equations with saturable nonlinearity. Sci. China Math. 2011, 54, 83-93. [CrossRef]

6. Zhou, Z.; Ma, D.F. Multiplicity results of breathers for the discrete nonlinear Schrödinger equations with unbounded potentials. Sci. China Math. 2015, 58, 781-790. [CrossRef]

7. Chen, P.; Tang, X.H. Existence of homoclinic orbits for $2 n$ th-order nonlinear difference equations containing both many advances and retardations. J. Math. Anal. Appl. 2011, 381, 485-505. [CrossRef]

8. Lin, G.H.; Zhou, Z. Homoclinic solutions in non-periodic discrete $\phi$-Laplacian equations with mixed nonlinearities. Appl. Math. Lett. 2017, 64, 15-20. [CrossRef]

9. Erbe, L.; Jia, B.G.; Zhang, Q.Q. Homoclinic solutions of discrete nonlinear systems via variational method. J. Appl. Anal. Comput. 2019, 9, 271-294. [CrossRef]

10. Zhang, Q.Q. Homoclinic orbits for discrete Hamiltonian systems with local super-quadratic conditions. Commun. Pure Appl. Anal. 2019, 18, 425-434. [CrossRef]

11. Lin, G.H.; Zhou, Z. Homoclinic solutions of discrete $\phi$-Laplacian equations with mixed nonlinearities. Commun. Pure Appl. Anal. 2018, 17, 1723-1747. [CrossRef]

12. Iannizzotto, A.; Tersian, S.A. Multiple homoclinic solutions for the discrete $p$-Laplacian via critical point theory. J. Math. Anal. Appl. 2013, 403, 173-182. [CrossRef]

13. Guiro, A.; Kone, B.; Ouaro, S. Weak homoclinic solutions of anisotropic difference equation with variable exponents. Adv. Differ. Equ. 2012, 2012, 154. [CrossRef]

14. Shi, H.P. Periodic and subharmonic solutions for second-order nonlinear difference equations. J. Appl. Math. Comput. 2015, 48, 157-171. [CrossRef]

15. Zhou, Z.; Yu, J.S.; Chen, Y.M. Periodic solutions of a $2 n$ th-order nonlinear difference equation. Sci. China Math. 2010, 53, 41-50. [CrossRef]

16. Mei, P.; Zhou, Z.; Lin, G.H. Periodic and subharmonic solutions for a $2 n$ th-order $\phi_{c}$-Laplacian difference equation containing both advances and retardations. Discret. Contin. Dyn. Syst. Ser. S 2019, 12, 2085-2095.

17. Avci, M.; Pankov, A. Nontrivial solutions of discrete nonlinear equations with variable exponent. J. Math. Anal. Appl. 2015, 431, 22-33. [CrossRef]

18. Lin, G.H.; Zhou, Z.; Yu, J.S. Ground state solutions of discrete asymptotically linear Schrödinger equations with bounded and non-periodic potentials. J. Dynam. Differ. Equ. 2020, 32, 527-555. [CrossRef]

19. Long, Y.H.; Wang, S.H. Multiple solutions for nonlinear functional difference equations by the invariant sets of descending flow. J. Differ. Equ. Appl. 2019, 25, 1768-1789. [CrossRef]

20. Long, Y.H.; Chen, J.L. Existence of multiple solutions to second-order discrete Neumann boundary value problems. Appl. Math. Lett. 2018, 83, 7-14. [CrossRef]

21. Zhou, Z.; Su, M.T. Boundary value problems for $2 n$-order $\phi_{c}$-Laplacian difference equations containing both advance and retardation. Appl. Math. Lett. 2015, 41,7-11. [CrossRef]

22. Zhou, Z.; Ling, J.X. Infinitely many positive solutions for a discrete two point nonlinear boundary value problem with $\phi_{c}$-Laplacian. Appl. Math. Lett. 2019, 91, 28-34. [CrossRef]

23. D'Aguì, G.; Mawhin, J.; Sciammetta, A. Positive solutions for a discrete two point nonlinear boundary value problem with $p$-Laplacian. J. Math. Anal. Appl. 2017, 447, 383-397. [CrossRef]

24. Bonanno, G.; Jebelean, P.; Şerban, C. Superlinear discrete problems. Appl. Math. Lett. 2016, 52, $162-168$. [CrossRef]

25. Bonanno, G.; Candito, P.; D’Aguì, G. Positive solutions for a nonlinear parameter-depending algebraic system. Electron. J. Differ. Equ. 2015, 2015, 1-14.

26. Bonanno, G.; Candito, P.; D'Aguì, G. Variational methods on finite dimensional banach spaces and discrete problems. Adv. Nonlinear Stud. 2014, 14, 915-939. [CrossRef]

27. Dinca, G.; Jebelean, P.; Mawhin, J. Variational and topological methods for Dirichlet problems with p-Laplacian. Port. Math. 2001, 58, 339-378.

28. Moghadam, M.K.; Avci, M. Existence results to a nonlinear $p(k)$-Laplacian difference equation. J. Differ. Equ. Appl. 2017, 23, 1652-1669.

29. Chen, Y.S.; Zhou, Z. Existence of three solutions for a nonlinear discrete boundary value problem with $\phi_{c}$-Laplacian. Symmetry 2020, 12, 1839. [CrossRef]

30. Heidarkhani, S.; Imbesi, M. Multiple solutions for partial discrete Dirichlet problems depending on a real parameter. J. Differ. Equ. Appl. 2015, 21, 96-110. [CrossRef] 
31. Galewski, M.; Orpel, A. On the existence of solutions for discrete elliptic boundary value problems. Appl. Anal. 2010, 89, 1879-1891. [CrossRef]

32. Imbesi, M.; Bisci, G.M. Discrete elliptic Dirichlet problems and nonlinear algebraic systems. Mediterr. J. Math. 2016, 13, 263-278. [CrossRef]

33. Bonanno, G.; Candito, P. Non-differentiable functionals and applications to elliptic problems with discontinuous nonlinearities. J. Differ. Equ. 2008, 244, 3031-3059. [CrossRef]

34. Bonanno, G.; Bisci, G.M. Infinitely many solutions for a boundary value problem with discontinuous nonlinearities. Bound. Value Probl. 2009, 2009, 1-20. [CrossRef]

Publisher's Note: MDPI stays neutral with regard to jurisdictional claims in published maps and institutional affiliations.

(C) 2020 by the authors. Licensee MDPI, Basel, Switzerland. This article is an open access article distributed under the terms and conditions of the Creative Commons Attribution (CC BY) license (http://creativecommons.org/licenses/by/4.0/). 